\section{Reply to Fe Talento et al}

To the Editor-We appreciate the comments by Fe Talento et $\mathrm{al}^{1}$ regarding our article evaluating the rate of effective empirical therapy for methicillin-resistant Staphylococcus aureus (MRSA) bloodstream infections.

Fe Talento et al $^{1}$ comment on the success of an integrated clinical microbiology service at Beaumont Hospital in Dublin, Ireland. This service is led by specially trained clinical microbiologists who communicate with the attending physician of each patient who has a positive blood culture result and provide recommendations for further evaluation and treatment. The authors note that, with use of this system, 80 of 83 patients with bloodstream infection due to MRSA received antibiotics. Of these 80 patients, $91 \%$ received appropriate antibiotics within the first 24 hours after the initial blood culture isolate was identified as suspected $S$. aureus. Three patients received no antibiotics and were not included in this calculation. Fe Talento and colleagues report that this rate is much higher than the rate of appropriate therapy reported in our study.

Although the success of this program is laudable, the authors' comparison is not accurate: $\mathrm{Fe}$ Talento et $\mathrm{al}^{1}$ judged appropriateness of treatment on the basis of antibiotics given after a blood culture result had first been noted to be positive and after $S$. aureus had been suspected as a pathogen (presumably after gram-positive cocci were identified as pathogens in the blood culture). In our study, appropriateness was judged on the basis of antibiotics administered on the day that the blood culture specimen was obtained-often days before the blood culture result was even known to be positive. For a more appropriate comparison, we suggest that $\mathrm{Fe}$ Talento and colleagues analyze the appropriateness of therapy on the day that blood samples for culture were obtained and not the day that a positive culture result was obtained.

We agree that communication between the microbiology laboratory and treating clinicians is an important tool to improve rates of effective antimicrobial therapy, and having an integrated clinical microbiology service is a wonderful asset (although it is probably not feasible in many community hospitals in the United States, the majority of which have less than 250 beds). Rapid diagnostic methods, such as polymerase chain reaction testing and culture on selective media (eg, CHROMagar; Becton Dickinson), are additional tools that can be used to assist with early identification of organisms once culture results turn positive to improve rates of effective antimicrobial therapy.

\section{ACKNOWLEDGMENTS}

Potential conflicts of interest. All authors report no conflicts of interest relevant to this article.
Carrie Herzke, MD; Luke F. Chen, MBBS, FRACP; Deverick J. Anderson, MD, MPH; Yong Choi, RN; Daniel J. Sexton, MD; Keith S. Kaye, MD, MPH

From the Johns Hopkins Medical Institute, Baltimore, Maryland (C.H.); Duke University Medical Center, Durham, North Carolina (L.F.C., D.J.A., Y.C., D.J.S.); and Wayne State University Medical Center, Detroit, Michigan (K.S.S.)

Address reprint requests to Carrie Herzke, MD, Johns Hopkins, 600 North Wolfe Street, Park 307, Baltimore, MD 21287 (cherzke1@jhmi.edu). Infect Control Hosp Epidemiol 2010; 31(9):983

(C) 2010 by The Society for Healthcare Epidemiology of America. All rights reserved. 0899-823X/2010/3109-0025\$15.00. DOI: $10.1086 / 656209$

\section{REFERENCE}

1. Fe Talento AF, Fitzpatrick F, Humphreys H, Smyth E. An integrated clinical microbiology service ensures optimal early antimicrobial therapy for methicillin-resistant Staphylococcus aureus bloodstream infection [letter]. Infect Control Hosp Epidemiol 2010;31(9):981-983 (in this issue).

\section{Inside-Out: The Changing Epidemiology of Methicillin-Resistant Staphylococcus aureus}

The increasing incidence of methicillin-resistant Staphylococcus aureus (MRSA) is a topic of concern in both the medical and the lay literature. ${ }^{1}$ Once thought of solely as a hospitalacquired pathogen, MRSA has been increasingly reported from the community, occurring in patients without established predisposing risk factors. Over the past decade, community-acquired MRSA (CA-MRSA) strains have been increasingly reported as the cause of serious infection and are now well recognized as a major cause of morbidity. ${ }^{2-4}$

The Veterans Affairs Medical Center (VAMC) in Washington, D.C., provided an ideal setting to study the changing epidemiology of MRSA, because the facility provides comprehensive emergency, outpatient, inpatient, and long-term care to a relatively closed population. Its electronic medical record allows infection control practitioners to monitor culture data facility-wide. We report the marked changes in the epidemiology of new clinical isolates of MRSA during the period 2001-2007.

During the 7 years of the study, approximately $40,000 \mathrm{pa}-$ tients received care at the medical center annually. We reviewed infection control data on clinical MRSA isolates recovered during the period 2001-2007. All new clinical isolates of MRSA were evaluated and categorized as either hospitalacquired MRSA (HA-MRSA) or CA-MRSA. We defined an isolate as HA-MRSA if an MRSA-positive culture result was obtained at least 48 hours after admission to the hospital for a person without obvious signs of infection at the time of admission; an isolate was also categorized as HA-MRSA if 
the patient had been in the facility, including the outpatient clinics, within 30 days before the onset of disease with MRSA. Isolates that did not fall under this definition were categorized as CA-MRSA.

Patients who were known to have MRSA infection or colonization were placed in contact isolation, in accordance with Centers for Disease Control and Prevention guidelines. The patients' electronic medical records were flagged, and healthcare workers were required to wear gloves and gowns for all procedures involving patient contact. A patient remained in isolation until infection at the primary site was no longer active. A hospital-wide hand hygiene program was in place throughout the study period, emphasizing the use of an alcohol-based hand disinfectant that had been proven to be effective at reducing the nosocomial acquisition of MRSA at the VAMC. ${ }^{5}$ During the period of this study, no active surveillance for MRSA was performed.

The number of new clinical MRSA isolates increased during the 7-year period, from 129 new MRSA isolates in 2001 to 221 new MRSA isolates in 2007. The number of HA-MRSA isolates decreased during this period, from 78 isolates in 2001 to 46 isolates in 2007. This decrease occurred despite a stable number of bed-days of care, so that the actual rate of HAMRSA infection decreased by approximately $41 \%$ from 2001 to 2007. In contrast, there was an almost 4-fold increase in the number of CA-MRSA isolates, from 41 isolates in 2001 to a peak of 183 isolates in $2005(P<.001)$ and a total of 175 isolates in 2007.

Figure 1 shows the dramatic increase in the proportion of isolates that were CA-MRSA from 2001 to 2006. During this 7 -year period, the proportion increased from $35 \%$ of all new clinical MRSA isolates in 2001 to $79 \%$ in 2007.

There was a shift in the location where the HA-MRSA isolates were recovered, from the inpatient wards to the outpatient clinical areas. The proportion of new MRSA clinical isolates recovered from the outpatient setting that were HAMRSA increased from $22 \%$ in 2001 to $50 \%$ in $2005-2007$ $(P<.001)$. There was also a shift in where the CA-MRSA clinical isolates were recovered. From 2001 to 2004, the majority of CA-MRSA clinical isolates were recovered from patients admitted to the inpatient wards; however, starting in 2005 , the greatest number of CA-MRSA clinical isolates were obtained from patients in the outpatient areas.

The integrated medical care system of the VAMC provides primary and specialized care to a stable patient base of approximately 40,000 veterans. Furthermore, veterans who receive their medical care in the VAMC tend not to use other healthcare facilities, thus offering an opportunity to understand the full spectrum of both HA-MRSA infection and CAMRSA infection in a large urban population. In addition, the fully integrated electronic medical record allowed infection control practitioners to track culture data and patient encounters from both the inpatient and outpatient settings in

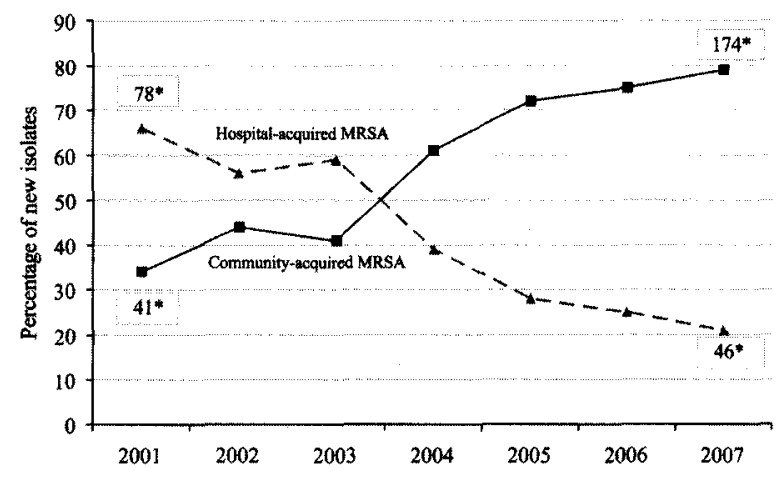

FIGURE 1. Proportions of new isolates of methicillin-resistant Staphylococcus aureus (MRSA) that were community acquired versus hospital acquired. *No. of new isolates.

a standardized manner, thus facilitating the ability to capture complete information on the entire patient population.

There have been many epidemiologic studies from institutions reporting increases in isolation of MRSA at their institutions. ${ }^{67}$ These authors, however, did not have the ability to study the epidemiology of CA-MRSA in their populations because of limited access of outpatient data. Because the VA provides comprehensive outpatient and inpatient care, we were able to show that the total number of new clinical isolates of MRSA increased during the 7-year study period and, importantly, that the driving force for this increase was a marked growth in the number of new clinical MRSA isolates acquired in the community. Furthermore, of the isolates that were acquired by patients in the hospital, more were recovered from persons in the outpatient clinics than from those in the inpatient wards.

We show clear evidence that MRSA is now being acquired predominately in the community and outpatient setting. We also demonstrated that the spread of HA-MRSA can be controlled using standard infection control practices without the aid of active surveillance, because the number of HA-MRSA isolates decreased during the 7 years of this study. A better understanding of the risk factors contributing to the significant increase in the incidence of MRSA infection in the community is needed, given the increasing number of people of all ages at risk for acquisition of this infection. Approaches to prevention, screening, and treatment need to reflect the changing epidemiology of this disease.

\section{ACKNOWLEDGMENTS}

Potential conflicts of interest. All authors report no conflicts of interest relevant to this article.

Leigh A. Kennedy, DO; Janet A. Gill, RN; Maureen E. Schultz, RN, MSN, CIC; Monica Irmler, RN; Fred M. Gordin, MD 
From the Infectious Diseases Section, Veterans Affairs Medical Center (L.A.K., J.A.G., M.E.S., M.I., F.M.G.), and the Department of Medicine, George Washington University (L.A.K., F.M.G.), Washington, DC.

Address reprint requests to Fred M. Gordin, MD, Infectious Diseases Section (151B); Veterans Affairs Medical Center, 50 Irving Street Northwest, Washington, DC 20422 (fred.gordin@va.gov).

Presented in part: the 45th Annual Meeting of the Infectious Diseases Society of America; San Diego, California; October 4-7, 2007.

Infect Control Hosp Epidemiol 2010; 31(9):983-985

(C) 2010 by The Society for Healthcare Epidemiology of America. All rights reserved. 0899-823X/2010/3109-0026\$15.00. DOI: 10.1086/655837

\section{REFERENCES}

1. Lowry FD. Staphylococcus aureus Infections. N Engl J Med 1998;339:520532.

2. Fridkin SK, Hageman JC, Morrison M, et al. Methicillin-resistant Staphylococcus aureus disease in three communities. N Engl J Med 2005;352: 1436-1445.

3. Kazakova SV, Hageman JC, Matava M, et al. A clone of methicillin-resistant Staphylococcus aureus among professional football players. $N$ Engl J Med 2005;352:468-475.

4. Chambers HF. The Changing Epidemiology of Staphlococcus aureus? Emerging Infect Dis 2001;7:178-182.

5. Gordin FM, Schultz ME, Huber RA, Janet JA. Reduction in nosocomial transmission of drug-resistant bacteria after introduction of an alcoholbased handrub. Infect Control Hosp Epidemiol 2005;26:650-653.

6. Seal JB, Moreira B, Bethel CD, Daum RS. Antimicrobial resistance in Staphylococcus aureus at the University of Chicago Hospitals: a 15-year longitudinal assessment in a large university-based hospital. Infect Control Hosp Epidemiol 2003;24:403-408.

7. Al-Tawfiq JA. Incidence and epidemiology of methicillin-resistant Staphylococcus aureus infection in a Saudi Arabian hospital, 1999-2003. Infect Control Hosp Epidemiol 2006;27:1137-1139.

\section{Pitfalls of Public Reporting}

To the Editor-Infection control is experiencing a muchneeded revolution, symbolized by a change in the name to "infection prevention." Active surveillance, public reporting, environmental decontamination, and bundles of best practices are among the significant developments that are causing an upheaval in our landscape.

Perhaps the most complex of these developments - and the one with the most profound implications-is public reporting. It was not too long ago that outcome data pertaining to hospital-acquired infections (HAIs) were deeply protected secrets, rarely escaping a hospital's firewall and often not even shared within an institution. Incomplete pictures of what was happening in hospitals might be discretely pinned to a poster board once per year or shared confidentially with the National Nosocomial Infection Surveillance system as a way of contributing to a national benchmark, but in almost all cases, the data were strictly for internal use by infection control personnel to inform their own decisions about how to assign priorities. Public reporting has changed all that, and the risks and benefits inherent in this approach are just beginning to be clarified. Two of the many potential pitfalls of public reporting include inadequate risk stratification and validation.

First, let us consider an example of inadequate risk stratification. The State of Vermont publicly reports rates of central line-associated bloodstream infections (CLABSIs) and selected surgical site infections (eg, infection after hysterectomies and total hip and knee arthroplasties). ${ }^{1}$ Vermont has only 1 tertiary care academic medical center, Fletcher Allen Health Care (FAHC); there is no other institution in the state for which a comparison of HAIs without risk stratification would be appropriate. A recent edition of Consumer Reports, ${ }^{2}$ which has more than 4 million subscribers, reported the top performers and "worst" hospitals in those states with publicly reported HAIs. FAHC was proclaimed to be the "bottom performer" in Vermont with respect to CLABSI rates, although it is noted that the hospital's CLABSI rate of 1.4 cases per 1,000 central line-days was $36 \%$ below the national average ("no, that's not a misprint"). The denominators for FAHC for this reporting period were 6,822 central line-days. Only 6 other hospitals in the state had enough data to meet the threshold for reporting, and the total denominator for all of these hospitals combined (2,573 central line-days) was less than one-half the size of FAHC alone. None of the other hospitals in the state reported a single CLABSI. Patients cared for in these small community hospital intensive care units with severe illness and risk factors for infection are frequently transferred to FAHC for more complex care, yet this measure is utterly devoid of risk stratification. Fortunately, there is no evidence that the lay public is using this information to decide in advance where to be critically ill, because one could imagine how intensive care provided elsewhere-in a small intensive care unit with no CLABSIs-could be associated with worse overall outcomes.

A more insidious and worrisome problem, however, is the lack of data validation. As HAI outcomes are released to the public, high rates will be bad for a hospital's business and reputation, independent of attempts to reduce reimbursement for care. In the past, infection control personnel were not discouraged from finding more infections, because the data were only for their own use. Now hospital administrators will bring significant pressure to bear on infection prevention programs, and rightfully so. High infection rates will provoke demands for explanations and action plans. A tremendous disincentive to report will arise, which could lead to measurement errors. This may not take the form of conscious underreporting but, rather, an unconscious, subtle laxity of surveillance or decisions not to report difficult to define events. Paradoxically, such a phenomenon could result in falling rates nationally and be cited as evidence of greater patient safety. As traditional paper medical records are replaced by enormously complex electronic records, it will be increasingly impractical for outside auditors unfamiliar with 